\title{
ON TOTALLY PARACOMPACT METRIC SPACES ${ }^{1}$
}

\section{A. LELEK}

A topological space is said to be totally paracompact [4] if every open basis contains a locally finite cover. It is known [2] that no reflexive infinite-dimensional Banach space is totally paracompact. It is also known [1], [3] that the space of irrationals with usual topology is not totally paracompact. In the present paper we prove a theorem which gives a necessary condition in order that a subset of a complete metric space be totally paracompact. This allows us to exhibit some pathology (Corollary 2) among the separable metric spaces which are not totally paracompact.

Let $\gamma$ be a collection of sets. We denote by $|\gamma|$ the union of elements of $\gamma$. The collection $\gamma$ is called point-finite if no point belongs to infinitely many elements of $\gamma$. By a Cantor set we mean a set homeomorphic with the Cantor ternary set.

Lemma. Let $X$ be a complete metric space and $Y \subset X \backslash Z$ where $Z$ is a nonempty subset of $X$ such that no point of $Z$ is isolated in $Z$. There exists an open basis $\beta$ of $Y$ such that if $\gamma \subset \beta$ is point-finite, then $X \backslash|\gamma|$ contains a Cantor set.

Proof. We take an increasing infinite sequence $A_{1} \subset A_{2} \subset \cdots$ of nonempty countable compact subsets $A_{n}$ of $Z$ such that no point of $A_{n}$ is isolated in $A_{n+1}$ for $n=1,2, \ldots$. Let $U_{n 1} \supset U_{n 2} \supset \ldots$ be open subsets of $X$ such that

$$
A_{n}=U_{n 1} \cap U_{n 2} \cap \cdots=\mathrm{Cl} U_{n 1} \cap \mathrm{Cl} U_{n 2} \cap \cdots
$$

and let $G_{n}(1), \cdots, G_{n}\left(k_{n}\right)$ be open subsets of $X$ such that

$$
A_{n} \subset G_{n}(1) \cup \cdots \cup G_{n}\left(k_{n}\right), \quad \operatorname{diam} G_{n}(i)<n^{-1}
$$

for $i=1, \cdots, k_{n}$. Let $V_{n}$ be an open subset of $X$ such that

$$
A_{n} \subset V_{n}, \quad \mathrm{Cl} V_{n} \subset G_{n}(1) \cup \ldots \cup G_{n}\left(k_{n}\right)
$$

and let $\beta_{n}^{\prime}$ denote the collection of all open subsets of $Y \backslash \mathrm{Cl} V_{n}$.

We put

$$
\beta_{n}=\beta_{n}^{\prime} \cup\left\{\left[G_{n}(i) \backslash \mathrm{Cl} U_{n j}\right] \cap Y \mid i=1, \cdots, k_{n} ; j=1,2, \cdots\right\}
$$

and we define $\beta=\beta_{1} \cup \beta_{2} \cup \ldots$. Since $Y \subset X \backslash A_{n}$, each point from

Received by the editors September 23, 1966.

1 This research had partially been done when the author was visiting the Louisiana State University and the University of Wisconsin. 
$Y \cap \mathrm{Cl} V_{n}$ belongs to a set $G_{n}(i) \backslash \mathrm{Cl} U_{n j}$ whose diameter is less than $n^{-1}$. Therefore $\beta$ is an open basis of $Y$.

Consider an arbitrary point-finite collection $\gamma \subset \beta$. The collection $\beta_{n} \backslash \beta_{n}{ }^{\prime}$ splits into $k_{n}$ increasing infinite sequences. Setting $\gamma_{n}=\beta_{n} \cap \gamma$ we conclude that $A_{n} \subset X \backslash \mathrm{Cl}\left|\gamma_{n}\right|$ for $n=1,2, \cdots$. No point of $A_{1}$ is isolated in $A_{2}$, and so there exist two points $p_{0}, p_{1}$ of $A_{2}$ belonging to $X \backslash \mathrm{Cl}\left|\gamma_{1}\right|$. We take an open neighborhood $W(m)$ of $p_{m}(m=0,1)$ such that

$$
W(m) \subset X \backslash \mathrm{Cl}\left|\gamma_{1}\right|, \quad \mathrm{Cl} W(0) \cap \mathrm{Cl} W(1)=\varnothing,
$$

and diam $W(m)<1$. Let us assume $n>1$ is an integer, and an open neighborhood $W=W\left(m_{1}, \cdots, m_{n-1}\right)$ of a point from $A_{n}$ is defined, where the indices $m_{1}, \cdots, m_{n-1}$ are 0 or 1 . Then there exist two points $q_{0}, q_{1}$ of $A_{n+1}$ belonging to $W \backslash \mathrm{Cl}\left|\gamma_{n}\right|$. We take an open neighborhood $W\left(m_{1}, \cdots, m_{n-1}, m\right)$ of $q_{m}(m=0,1)$ such that

$$
\begin{aligned}
& \mathrm{Cl} W\left(m_{1}, \cdots, m_{n-1}, m\right) \subset W\left(m_{1}, \cdots, m_{n-1}\right) \backslash \mathrm{Cl}\left|\gamma_{n}\right|, \\
& \mathrm{Cl} W\left(m_{1}, \cdots, m_{n-1}, 0\right) \cap \mathrm{Cl} W\left(m_{1}, \cdots, m_{n-1}, 1\right)=\varnothing,
\end{aligned}
$$

and diam $W\left(m_{1}, \cdots, m_{n-1}, m\right)<n^{-1}$. The intersection

$$
C=\bigcap_{n=1}^{\infty} \underset{\left(m_{1}, \cdots, m_{n}\right)}{\bigcup} \mathrm{Cl} W\left(m_{1}, \cdots, m_{n}\right)
$$

is a Cantor set, and $C \subset X \backslash\left(\left|\gamma_{1}\right| \cup\left|\gamma_{2}\right| \cup \ldots\right)=X \backslash|\gamma|$.

Theorem. Let $X$ be a complete metric space and $Y \subset X$. If $Y$ is totally paracompact and $p \in X \backslash Y$, then either $X \backslash Y$ contains a Cantor set containing $p$ or each subset of $X \backslash Y$ containing $p$ has an isolated point.

Proof. Suppose there exists a set $Z \subset X \backslash Y$ such that $p \in Z$ and no point of $Z$ is isolated in $Z$. Applying the lemma to the sets

$$
\begin{aligned}
& X_{n}=\left\{x \mid \operatorname{dist}(p, x) \leqq n^{-1}\right\}, \\
& Y_{n}=X_{n} \cap Y, \quad Z_{n}=\text { Int } X_{n} \cap Z,
\end{aligned}
$$

we get a Cantor set $C_{n} \subset X_{n} \backslash Y_{n}$ because $Y_{n}$ is totally paracompact $(n=1,2, \cdots)$. The union $\{p\} \cup C_{1} \cup C_{2} \cup \cdots$ is a Cantor set contained in $X \backslash Y$.

Corollary 1. Let $X$ be a complete separable metric space and $Y \subset X$. If $Y$ is uncountable and $Y$ contains no Cantor set, then $X \backslash Y$ is not totally paracompact.

Each closed subset of a totally paracompact space is totally paracompact. Thus if $X$ is totally paracompact, no closed subset of $X$ is 
homeomorphic with the irrationals. Answering a question raised by E. Michael we prove the inverse implication is not true. Namely, as a result of Bernstein's construction [5, p. 422] which uses the axiom of choice one can obtain a subset $X$ of the Euclidean $(n+1)$-space $E^{n+1}$ such that both $X$ and $E^{n+1} \backslash X$ have the cardinality of the continuum and contain no Cantor sets.

COROLlary 2. If the axiom of choice is true, then there exists, for every $n \geqq 0$, an $n$-dimensional subset $X$ of the Euclidean $(n+1)$-space such that $X$ is not totally paracompact and $X$ contains no Cantor set.

Observe that an analogous singularity exists among totally paracompact spaces. In fact, as a result of Lusin's construction [5, pp. 432-433] using the axiom of choice and the continuum hypothesis one gets an uncountable subset $X$ of the irrationals such that $X$ is totally paracompact and $X$ contains no Cantor set.

\section{BIBLIOGRAPHY}

1. A. V. Arhangel'skiř, On the metrization of topological spaces, Bull. Acad. Polon. Sci. Sér. Sci. Math. Astronom. Phys. 8 (1960), 589-595.

2. H. H. Corson, Collections of convex sets which cover a Banach space, Fund. Math. 49 (1961), 143-145.

3. H. H. Corson, T. J. McMinn, E. A. Michael, and J.-I. Nagata, Bases and local finiteness, Notices Amer. Math. Soc. 6 (1959), 814.

4. R. M. Ford, Basis properties in dimension theory, Doctoral Dissertation, Auburn University, Auburn, Ala., 1963.

5. C. Kuratowski, Topologie. I, Państwowe Wydawnictwo Naukowe, Warsaw, 1958.

Institute of Mathematics of the Polish Academy of Sciences 